\title{
Sterile leukocyturia affects urine neutrophil gelatinase-associated lipocalin concentration in type 2 diabetic patients
}

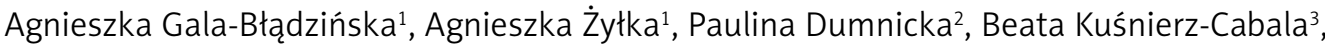 \\ Magdalena Barbara Kaziuk ${ }^{4}$, Marek Kuźniewski ${ }^{4}$
}

\begin{abstract}
'Department of Internal Medicine, Nephrology and Endocrinology, St Queen Jadwiga Clinical District Hospital No. 2, Rzeszow, Poland

${ }^{2}$ Department of Medical Diagnostics, Jagiellonian University Medical College, Krakow, Poland

${ }^{3}$ Department of Diagnostics, Chair of Clinical Biochemistry, Jagiellonian University Medical College, Krakow, Poland

${ }^{4}$ Department of Nephrology, Jagiellonian University Medical College, Krakow, Poland
\end{abstract}

Submitted: 1 August 2015

Accepted: 27 October 2015

Arch Med Sci 2017; 13, 2: 321-327

DOI: https://doi.org/10.5114/aoms.2016.64043

Copyright @ 2016 Termedia \& Banach

\section{Abstract}

Introduction: Increased urine neutrophil gelatinase-associated lipocalin ( $U N G A L)$ concentrations are associated with the early phase of kidney damage. Urine NGAL may increase due to production by neutrophils present in urine, particularly in patients with urinary tract infections. The aim of the study was to assess the relationship between sterile leukocyturia and UNGAL concentrations in patients with type 2 diabetes (DMt2) at early stages of diabetic kidney disease.

Material and methods: The study included $115 \mathrm{DMt} 2$ patients aged 60.0 \pm 15.5 years, with albuminuria $<300 \mathrm{mg} / \mathrm{g}$ creatinine and estimated glomerular filtration rate $\geq 60 \mathrm{ml} / \mathrm{min} / 1.73 \mathrm{~m}^{2}$ prospectively recruited at the nephrology ambulatory clinic in 2014-2015. The exclusion criteria were urinary tract infections (excluded by urine culture) and other diseases influencing UNGAL, including inflammatory and other kidney diseases. Urine concentrations of NGAL, albumin and creatinine were measured in the first morning samples, and the urine albumin to creatinine ratio (UACR) and UNGAL to creatinine ratio (UNCR) were calculated. Leukocyturia was detected microscopically.

Results: Sterile leukocyturia was present in 15\% (95\% confidence interval: 9-23\%) of patients. Patients with leukocyturia had higher UNGAL and UNCR than patients without leukocyturia. In(UNCR) correlated with $\operatorname{In}(\mathrm{UACR})$ in the whole group $(R=0.59 ; p<0.001)$ and in patients without leukocyturia $(R=0.56 ; p<0.001)$. In multiple regression, age, $\ln (\mathrm{UACR}), \ln \left(\mathrm{HbA}_{1 c}\right)$ and leukocyturia were independent positive predictors of $\ln (\mathrm{UNCR})$. Among patients with leukocyturia, the associations of UNCR with UACR, age and $\mathrm{HbA}_{1 c}$ were non-significant.

Conclusions: In patients with DMt2, the presence of sterile leukocyturia coexists with increased UNGAL and UNCR. Leukocyturia interferes with the relationship between UNCR and UACR or $\mathrm{HbA}_{1 \mathrm{c}}$.

Key words: diabetic nephropathy, neutrophil gelatinase-associated lipocalin, tubular injury biomarkers.

\author{
Corresponding author: \\ Agnieszka Gala-Błądzińska \\ PhD \\ Department \\ of Internal Medicine, \\ Nephrology and \\ Endocrinology \\ St Queen Jadwiga \\ Clinical District Hospital No. 2 \\ 60 Lwowska St \\ 35-301 Rzeszow, Poland \\ Phone: +48 604416170 \\ +48 178664045 \\ Fax: +48 178664048 \\ E-mail: bladzinscy@wp.pl
}




\section{Introduction}

Despite the progress in diagnosis and treatment of diabetic kidney disease (DKD), DKD remains the most frequent cause of chronic kidney disease (CKD) including CKD stage 5, or renal failure [1]. According to a report of the United States Renal Data System published in 2014, patients with DKD constitute nearly $45 \%$ of patients undergoing renal replacement therapy [1]. Diabetic kidney disease is frequently attributed to glomerular structural changes (enlargement of the mesangial space, thickening of the basement membrane), as well as damage and loss of podocytes, associated with nodular glomerulosclerosis (Kimmelstiel-Wilson nodular disease) [2]. However, data accumulating from the 1990s suggest that not only changes in glomeruli, but also changes in renal tubules and in the interstitial tissue, play an important role in the development of DKD, especially in type 2 diabetes (DMt2) [3].

Neutrophil gelatinase-associated lipocalin (NGAL) is a $25 \mathrm{kDa}$ protein, initially found in activated neutrophils, where it is covalently bound to human neutrophil gelatinase. A NGAL is a secretory protein belonging to the lipocalin family. Physiologically, it is expressed at low levels in the kidneys, trachea, lungs, stomach and colon [4]; mean urine NGAL concentrations in healthy individuals were reported to be about $20 \mathrm{ng} / \mathrm{ml}$ [5]. In the kidney, NGAL is filtered through the glomeruli and then almost completely absorbed by the proximal tubules in the process of megalin-dependent endocytosis. The concentrations of NGAL in urine (UNGAL) may thus increase due to impaired reabsorption in proximal tubules. Nevertheless, the main fraction of UNGAL comes from the distal tubule cells that synthesize the protein in response to various insulting factors $[4,6]$. Highly increased UNGAL concentrations (> 200-300 $\mathrm{ng} / \mathrm{ml}$ ) are observed in patients with acute kidney injury [7]; however, the protein is also considered a marker of tubular dysfunction in CKD [8]. It was observed that the concentrations of both NGAL and other metalloproteinases in serum are higher in CKD patients, particularly in hemodialysis patients $[9,10]$.

Several studies have shown that UNGAL is higher among patients with leukocyturia caused by infections of the urinary tract $[11,12]$. However, we have found no studies investigating the impact of sterile leukocyturia on the values of $\mathrm{UN}$ GAL and urine NGAL to creatinine ratio (UNCR) in DMt2 patients.

The aim of this study was to investigate the association of sterile leukocyturia with UNGAL concentrations and UNCR among DMt2 patients at early stages of DKD. Also, we studied the associations of UNGAL and UNCR with clinically important patients' characteristics, including sex, age, body mass index (BMI), diabetes duration, glycohemoglobin $\left(\mathrm{HbA}_{1 \mathrm{c}}\right)$, estimated glomerular filtration rate (eGFR), albuminuria, serum cholesterol and triglyceride concentrations.

\section{Material and methods}

\section{Patients}

The observational study involved DMt2 patients, aged 18 and above, with eGFR above $60 \mathrm{ml}$ $\min / 1.73 \mathrm{~m}^{2}$, urine albumin to creatinine ratio $(U A C R)<300 \mathrm{mg} / \mathrm{g}$, and without urinary tract infections, prospectively recruited between November 2014 and August 2015 at the ambulatory clinic of the Nephrology Department of a clinical hospital (St Queen Jadwiga District Hospital No. 2 in Rzeszow, Poland). Diabetes mellitus type 2 was diagnosed in accordance with the recommendations of the American Diabetes Association [13]. Urinary tract infections were excluded by the interview and urine examination, including urine culture. Other exclusion criteria were: treatment with potentially nephrotoxic agents, kidney diseases other than DKD, cancer, documented allergy or autoimmune disease, anemia, unstable hypertension, pregnancy and acute disease or inflammation.

The control group included adult non-diabetic patients, with eGFR $>60 \mathrm{ml} / \mathrm{min} / 1.73 \mathrm{~m}^{2}$, without proteinuria, leukocyturia or urinary tract infection, who attended the control visits at the Ambulatory Clinic of the Nephrology Department due to stable hypertension or benign simple kidney cyst. The exclusion criteria were the same as for the study group. The control group was meant solely to provide an insight into UNGAL concentration and UNCR values among non-diabetic subjects with age, sex and co-morbidities similar to those observed in the studied diabetic patients. The reference ranges for UNGAL are provided by the manufacturer of the test, and the aim of including the control group was only to verify this information; therefore the control group was not planned to be large.

The study was conducted according to the Declaration of Helsinki and its protocol was approved by the Bioethical Committee of the Polish Chamber of Physicians (approval number 40/2013/B). All the participants gave written informed consent for the study.

\section{Laboratory tests}

The laboratory tests were conducted at the Clinical Department of Laboratory Diagnostics of St. Queen Jadwiga District Hospital No. 2 in Rzeszow, Poland. All tests were performed on the day of sample collection.

The first morning samples of urine were collected using the midstream collection method into sterile containers and were used for urine culture, 
general urine examination, and measurements of NGAL, albumin, and creatinine concentrations. Blood samples for laboratory tests were collected after an overnight fast. The $\mathrm{HbA}_{1 \mathrm{c}}$ was measured in $\mathrm{K}_{2}$ EDTA-anticoagulated whole blood. The concentrations of glucose, C-reactive protein, creatinine, triglycerides (TG), total cholesterol, low-density lipoprotein (LDL) cholesterol and high-density lipoprotein (HDL) cholesterol were measured in serum. White blood cell count was performed with an ADVIA 2120i Siemens 5-diff hematological analyzer (Siemens Healthcare Diagnostic, Germany).

The general urine examination was carried out using the Labumat-Urised 2 analyzer and microscopic examination of urine sediment. Urinary tract infection was excluded by urine culture in patients with leukocyturia, i.e. 10 or more leukocytes per high power field in urine sediment examination. The concentration of UNGAL was measured using the chemiluminescent microparticle immunoassay (ARCHITECT, Abbott Diagnostics, Abbott Park, USA). Urine albumin concentrations were measured with the immunoturbidimetric assay, and urine creatinine with the enzymatic method with creatinine and creatinine using an Olympus AU680 Immunochemistry analyzer. The UACR was calculated as urine albumin [mg/l]/urine creatinine $[\mathrm{g} / \mathrm{l}]$ and UNCR as UNGAL $[\mu \mathrm{g} / \mathrm{l}] /$ urine creatinine $\left[\mathrm{g} / \mathrm{ll}\right.$. The $\mathrm{HbA}_{1 \mathrm{c}}$ was measured with the immunochromatographic method. The GFR values were estimated using the 2009 CKD-EPI equation, which was recommended as the standard way for determining eGFR [14].

\section{Statistical analysis}

Data for categories are shown as number of patients and percentage of the appropriate group, with $95 \%$ confidence interval $(95 \% \mathrm{Cl})$ for the proportion (calculated using the Wilson procedure with a correction for continuity). Mean \pm standard deviation or median (25-75 percentile) is shown for continuous variables with or without normal distribution (as tested with the Shapiro-Wilk test). Groups were compared using the $\chi^{2}$ test, one-way analysis of variance (ANOVA) or Kruskal-Wallis ANOVA, with Bonferroni's post-hoc tests (the differences in post-hoc tests were considered significant at $p \leq 0.016)$. The Mann-Whitney test or $t$-test was used for comparisons between 2 groups. Simple correlations were studied using Pearson's coefficient, after log-transformation (normal logarithm) of right-skewed variables. Linear multiple regression was calculated using predictors that were significantly or nearly significantly $(p \leq 0.1)$ correlated with the dependent variable in simple regression. Results were considered significant at $p \leq 0.05$. Statistica 10.0 (StatSoft, Tulsa, USA) software was used for computations.

\section{Results}

Among 115 diabetic patients studied, 17 (15\%; 95\% Cl: 9-23\%) had sterile leukocyturia. Diabetic patients with leukocyturia had significantly higher urine NGAL and albumin (both non-standardized concentrations and the values normalized to urine creatinine) than diabetic patients without leukocyturia and non-diabetic subjects (Table I). Serum creatinine concentrations and eGFR values did not differ between the groups. The percentage of women among patients with leukocyturia was significantly higher than among subjects without leukocyturia (Table I).

Urine NGAL concentrations were significantly correlated with urine albumin concentrations (log-transformed variables) in the whole group of diabetic patients $(R=0.59 ; p<0.001)$ and in patients without leukocyturia $(R=0.57 ; p<0.001)$; the same associations were found between UNCR and UACR (Figure 1). However, these correlations were non-significant in diabetic patients with leukocyturia (Figure 1).

Diabetic women had higher UNGAL and UNCR than diabetic men: 18.3 (8.7-44.8) vs. 14.2 (6.1$24.8) \mathrm{ng} / \mathrm{ml} ; p=0.035$ and 28.1 (9.0-65.7) vs. 11.3 (3.3-18.0) $\mu \mathrm{g} / \mathrm{g} ; p<0.001$, respectively. Among patients without leukocyturia, only UNCR was significantly associated with sex (Figure 2 ). In the control group, there were no significant differences between sexes regarding UNGAL, which was non-significantly higher in men (20.1 (7.4-38.4) vs. $8.3(5.0-20.1)(p=0.1))$, and UNCR, which was non-significantly higher in women: 12.5 (6.0-28.3) vs. $7.7(5.5-13.7) \mu \mathrm{g} / \mathrm{g}(p=0.3)$. There were no sex-related differences regarding urine albumin or UACR, both in diabetic patients and non-diabetic controls.

In diabetic patients without leukocyturia, In(UNCR) was significantly correlated with age $(R=0.22 ; p=0.028)$; there was no such correlation for $\ln (U N G A L)$. Urine NGAL and UNCR were not correlated with the duration of diabetes, and there were no differences between patients with newly diagnosed diabetes compared to the rest of the group, irrespectively of leukocyturia. However, both $\ln (U N G A L)$ and $\ln (U N C R)$ were significantly associated with $\ln \left(\mathrm{HbA}_{1 \mathrm{c}}\right)$ in the whole group of diabetic patients $(R=0.34 ; p=0.003$ and $R=0.30$; $p=0.008$, respectively) and in subjects without leukocyturia $(R=0.46$; $p<0.001$ and $R=0.44$; $p<0.001)$. The correlations were not significant in patients with leukocyturia $(R=-0.02 ; p=0.9$ and $R=-0.12 ; p=0.7$, respectively).

There were no correlations between UNGAL or UNCR and BMI. Also, UNGAL and UNCR did not correlate with eGFR in our group of diabetic patients, irrespectively of leukocyturia ( $p$-values $\geq 0.2$ ). We noted no correlations of UNGAL and UNCR with 
Table I. Clinical characteristics of the groups and laboratory data

\begin{tabular}{|c|c|c|c|c|}
\hline \multirow[t]{2}{*}{ Parameter } & \multirow{2}{*}{$\begin{array}{l}\text { Non-diabetic } \\
\text { patients without } \\
\text { leukocyturia } \\
(N=21)\end{array}$} & \multicolumn{2}{|c|}{ Diabetic patients } & \multirow[t]{2}{*}{$P$-value* } \\
\hline & & $\begin{array}{l}\text { Without } \\
\text { leukocyturia } \\
(N=98)\end{array}$ & $\begin{array}{c}\text { With } \\
\text { leukocyturia } \\
(N=17)\end{array}$ & \\
\hline Female sex, $n(\% / 95 \% \mathrm{Cl})$ & $11(52 / 30-74)^{a}$ & $45(46 / 36-56)^{\mathrm{b}}$ & $16(94 / 69-100)^{a, b}$ & 0.001 \\
\hline Age [years] & $56.6 \pm 14.9$ & $62.1 \pm 12.4$ & $59.2 \pm 18.5$ & NS \\
\hline $\mathrm{BMI}\left[\mathrm{kg} / \mathrm{m}^{2}\right]$ & $27.8 \pm 5.3^{c}$ & $31.8 \pm 5.8^{c}$ & $32.0 \pm 5.5$ & 0.017 \\
\hline Diabetes duration [years] & - & $6(2-10)$ & $10(5-15)$ & $\mathrm{NS}^{\mathrm{S}}$ \\
\hline Newly diagnosed diabetes, $n(\% / 95 \% \mathrm{Cl})$ & - & $19(19 / 12-29)$ & $2(12 / 2-38)$ & $N S^{5}$ \\
\hline $\mathrm{HbA}_{1 \mathrm{c}}(\%)$ & - & $6.5(5.9-8.6)$ & $6.7(6.2-8.2)$ & $\mathrm{NS}^{5}$ \\
\hline $\mathrm{HbA}_{1 \mathrm{c}}[\mathrm{mmol} / \mathrm{mol}]$ & - & $48(41-70)$ & $50(44-66)$ & $\mathrm{NS}^{5}$ \\
\hline Total cholesterol [mmol/l] & $5.13(4.47-6.97)$ & $4.76(3.90-5.77)$ & $5.42(4.33-6.22)$ & NS \\
\hline HDL cholesterol [mmol/l] & $3.97(2.96-5.13)^{c}$ & $2.84(1.99-3.75)^{c}$ & $3.05(2.40-4.01)$ & 0.007 \\
\hline LDL cholesterol [mmol/l] & $1.27(1.18-1.49)$ & $1.19(0.96-1.42)$ & $1.24(1.01-1.47)$ & NS \\
\hline Triglycerides $[\mathrm{mmol} / \mathrm{l}]$ & $1.51(0.85-1.99)$ & $1.63(1.26-2.13)$ & $2.51(1.23-3.17)$ & NS \\
\hline Serum creatinine $[\mu \mathrm{mol} / \mathrm{l}]$ & $71.6(70.7-79.6)^{\mathrm{a}}$ & $68.1(60.1-78.7)$ & $61.9(53.0-68.1)^{\mathrm{a}}$ & 0.008 \\
\hline eGFR (CKD-EPI) [ml/min/1.73 $\left.\mathrm{m}^{2}\right]$ & $84(77-99)$ & $93(79-100)$ & $88(81-98)$ & NS \\
\hline Urine creatinine $[\mathrm{g} / \mathrm{l}]$ & $1.39(0.71-2.24)$ & $1.04(0.69-1.62)$ & $0.86(0.60-1.44)$ & NS \\
\hline Urine NGAL $[\mu \mathrm{g} / \mathrm{l}]$ & $10.5(6.0-24.6)^{\mathrm{a}}$ & $13.7(6.1-22.0)^{\mathrm{b}}$ & $72.2(45.6-133.0)^{a, b}$ & $<0.001$ \\
\hline UNCR $[\mu \mathrm{g} / \mathrm{g}]$ & $11.9(5.9-15.9)^{\mathrm{a}}$ & $11.4(5.4-26.8)^{b}$ & $88.4(65.7-198.8)^{a, b}$ & $<0.001$ \\
\hline Urine albumin $[\mathrm{mg} / \mathrm{l}]$ & $5.7(3.0-12.0)^{\mathrm{a}}$ & $6.8(3.1-13.2)^{b}$ & $25.1(11.4-36.8)^{a, b}$ & 0.006 \\
\hline UACR $[\mathrm{mg} / \mathrm{g}]$ & $4.7(3.6-8.7)^{\mathrm{a}}$ & $5.8(3.1-15.1)^{\mathrm{b}}$ & $16.1(7.8-50.1)^{\mathrm{a}, \mathrm{b}}$ & 0.002 \\
\hline Hypertension, $n(\% / 95 \% \mathrm{Cl})$ & $14(67 / 43-85)$ & $78(80 / 70-87)$ & $13(76 / 50-92)$ & NS \\
\hline Ischemic heart disease, $n(\% / 95 \% \mathrm{Cl})$ & $2(10 / 2-32)$ & $22(22 / 15-32)$ & $1(6 / 0-31)$ & NS \\
\hline Heart failure, $n(\% / 95 \% \mathrm{Cl})$ & $2(10 / 2-32)$ & $8(8 / 4-16)$ & $1(6 / 0-31)$ & NS \\
\hline $\begin{array}{l}\text { RAA blockade (ACEI or AT1-blockers), } \\
n(\% / 95 \% \mathrm{CI})\end{array}$ & $11(52 / 30-74)$ & $67(68 / 58-77)$ & $14(82 / 56-95)$ & NS \\
\hline Statin, $n(\% / 95 \% \mathrm{Cl})$ & $10(48 / 26-70)$ & $51(52 / 42-62)$ & $7(41 / 19-67)$ & NS \\
\hline
\end{tabular}

serum lipid concentrations, except for a weak negative correlation between $\mathrm{HDL}$-cholesterol and $\ln (\mathrm{UNGAL})$ in patients without leukocyturia $(R=-0.25 ; p=0.021)$, and a similar correlation with $\ln (\mathrm{UNCR})$ in patients with leukocyturia $(R=-0.51 ; p=0.050)$. There were no differences in UNGAL or UNCR between patients with and without hypertension. Also, treatment with statin or RAA blockade was not associated with significant differences in UNGAL concentrations or UNCR values.

In multiple regression (Table II), In(urine albumin), $\ln \left(\mathrm{HbA}_{1 c}\right)$ and leukocyturia significantly predicted $\ln (\mathrm{UNGAL})$, whereas age, $\ln (\mathrm{UACR})$, $\ln \left(\mathrm{HbA}_{1 \mathrm{c}}\right)$ and leukocyturia were independent predictors of $\ln (\mathrm{UNCR})$.

\section{Discussion}

In the studied group of DMt2 patients with stage 1 and 2 CKD, the presence of leukocyturia was an independent predictor for UNGAL and UNCR concentrations. Urine NGAL and UNCR values were significantly higher in patients with leukocyturia than in patients without leukocyturia. In fact, UNGAL and UNCR in DMt2 patients without leukocyturia did not differ significantly from the control group, which may reflect the impact of co- 


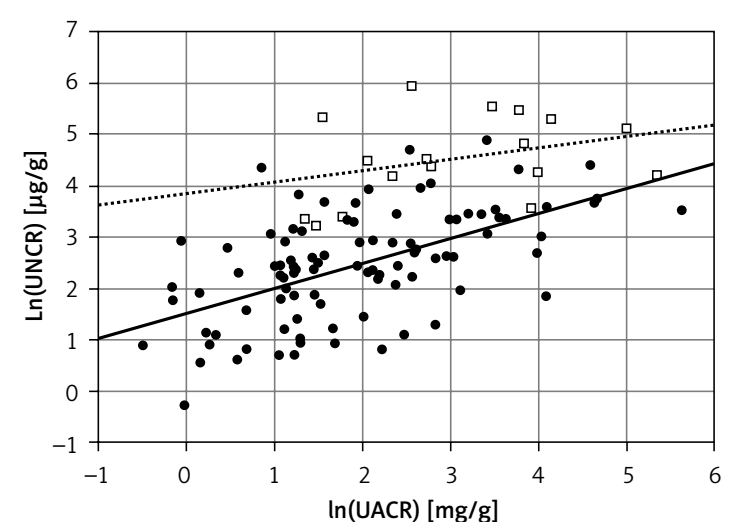

Figure 1. Relationship between UACR and UNCR in diabetic patients without leukocyturia (black dots, solid line) and with leukocyturia (open squares, dotted line). Correlation coefficients and $p$-values are shown for the whole group of diabetic patients, subjects without leukocyturia and subjects with leukocyturia

morbidities, e.g. hypertension. Leukocytes may be the source of UNGAL in patients with leukocyturia. The leukocytes present in urine may derive from all parts of the urinary tract; however, the routine laboratory tests do not allow for identification of the source of leukocyturia. Determining the cause of leukocyturia requires a detailed interview, thorough physical examination including gynecological and urological consultation, as well as imaging or endoscopic examination of the urinary tract. In our study, patients with leukocyturia and positive urine cultures were excluded [15].

However, sterile leukocyturia in studied patients may also be associated with the injury of kidney tubular epithelial cells and increased expression of NGAL in tubular cells. Ding et al. [8] found that the increase of NGAL is an early biomarker of tubular epithelial injury in IgA nephropathy. Also, several authors support the tubulo-centric theory of DKD in a group of patients with DMt2. Clinical observations suggest that hyperglycemia, atherogenic dyslipidemia, obesity or hypertension, all commonly observed in DMt2, may lead

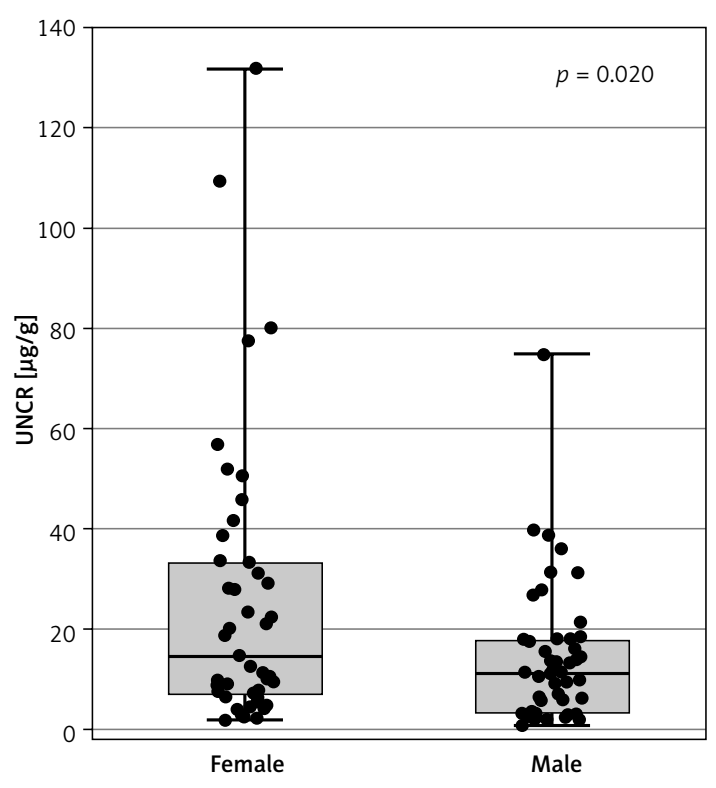

Figure 2. Gender-related differences in UNCR among diabetic patients without leukocyturia

Data are shown as median, $25^{\text {th }}-75^{\text {th }}$ percentile (boxes), and range (whiskers); points represent row data.

to the development of an inflammatory state in the tubular-epithelial tissue, increased production of extracellular matrix, and the epithelial mesenchymal transition of renal tubular cells $[8,16]$. Due to the inflammatory state, the tubular-epithelial tissue becomes infiltrated by leukocytes and monocytes, which, being transformed into tissue macrophages, induce reparatory changes and fibrosis. Because of this process, leading to irreversible kidney failure, DKD can be considered an inflammatory disease $[17,18]$. The recommended therapy in these patients includes, inter alia, lipid-lowering and antihypertensive therapy [14, 19], anti-diabetic drugs [20], and lifestyle changes to reduce overweight and obesity [21].

The present study has shown that in DMt2 patients, albuminuria correlated independently with higher values of UNGAL and UNCR. The increased excretion of albumin in the urine remains a recog-

Table II. Multiple regression to predict UNGAL concentrations and UNCR in DMT2 patients

\begin{tabular}{|lcccc|}
\hline Independent variable & \multicolumn{2}{c|}{$\ln ($ UNGAL) } & \multicolumn{2}{c|}{$\ln ($ UNCR) } \\
\cline { 2 - 5 } & Beta \pm SE & $P$-value & Beta \pm SE & $P$-value \\
\hline Age & - & - & $0.25 \pm 0.09$ & 0.006 \\
\hline Female sex & $0.07 \pm 0.09$ & 0.4 & $0.14 \pm 0.08$ & 0.078 \\
\hline In(HbA $\left.{ }_{1 c}\right)$ & $0.18 \pm 0.09$ & 0.039 & $0.31 \pm 0.09$ & 0.001 \\
\hline HDL-C & $-0.15 \pm 0.08$ & 0.068 & - & - \\
\hline In(urine albumin) & $0.39 \pm 0.09$ & $<0.001$ & $0.28 \pm 0.09$ & 0.003 \\
\hline In(UACR) & - & - & $0.46 \pm 0.08$ & $<0.001$ \\
\hline Leukocyturia & $0.45 \pm 0.09$ & $<0.001$ & $R^{2}=0.59$ & $<0.001$ \\
\hline Whole model & $R^{2}=0.56$ & $<0.001$ & & - \\
\hline
\end{tabular}

HDL-C-HDL cholesterol. 
nized risk factor for CKD [13, 22]. This observation is consistent with other studies [23, 24]. The present study indicates, however, that the presence of leukocyturia interferes with this positive correlation between UNGAL and albuminuria (it is not significant among patients with leukocyturia). It may be a consequence of the relatively low number of patients with leukocyturia in this study. However, if the increased number of leukocytes in urine is associated with tubular cells' injury, it may be assumed that this group of patients has an increased predisposition for tubulopathy rather than glomerulopathy in the course of DMt2. The group with DMt2 and sterile leukocyturia may presumably develop the clinical phenotype of the disease without progression to severely increased albuminuria [25]. Consistently, in the present study, UNGAL and UNCR were not correlated with DMt2 duration. Thus, in some patients, UNGAL and UNCR seem more promising and earlier markers of DMt2-associated kidney disease than UACR. However, this issue requires further prospective studies recruiting much higher numbers of DMt2 patients.

The present study did not show a significant correlation between eGFR and UNGAL or UNCR, in contrast to the negative correlation described in several publications [23, 26, 27]. However, the study groups differed from those of Bolignano et al. [23], and the method used for eGFR calculation differed from the one used by Yang et al. [27]. This may in part explain the discrepancy.

In the studied DMt2 patients, sterile leukocyturia coinciding with high UNGAL and UNCR was observed predominantly in women. Even among DMt2 patients without leukocyturia, the highest UNCR values were generally seen in females than in males. These observations may reflect higher susceptibility to tubular kidney injury in the course of DMt2 among women with CKD stages G1-G2/A1-A2. This corresponds to the results of the UKPDS study, indicating that even in the absence of albuminuria women with DMt2 are more prone to the development of progressive kidney injury in the course of DMt2 [28]. Also, in the study of Cullen et al. [11], 95 th percentiles for UNGAL and UNCR were higher in women.

Additionally, a weak but independent correlation of UNGAL and UNCR with $\mathrm{HbA}_{1 c}$ was observed in patients with DMt2. There was, however, no such correlation in a subgroup of patients with leukocyturia. In the study by Nielsen et al. [24], including 177 patients with eGFR and albuminuria (a sample comparable to those studied here), a similar positive correlation of $\mathrm{HbA}_{1 c}$ and UNCR was observed. Nevertheless, these authors provide no information on the prevalence of leukocyturia in their patients. Finally, in the present study, there was a positive correlation between age and
UNCR. The presence of leukocyturia rendered this correlation statistically insignificant, which may result from the relatively low number of patients with leukocyturia. Another hypothesis is that in some DMt2 patients, renal tubules are prone to early damage independently of age.

In summary, it appears that the presence of leukocyturia does not eliminate the possibility to use UNGAL and UNCR for the assessment of kidney tubular injury. However, leukocyturia should be taken into account when interpreting the results. In patients with acute kidney injury and coexisting leukocyturia, Cullen et al. [11] suggested a correction of the cut-off value for UNGAL. In turn, Decavele et al. [12] proposed a formula to correct UNGAL concentrations depending on numbers of urinary leukocytes in patients with urinary tract infections. The issue of UNGAL corrections in patients with DKD remains open.

In conclusion, the presence of sterile leukocyturia was observed in $15 \%$ of DMt2 patients, mainly women. The presence of leukocyturia in this group requires a thorough differential diagnostics, taking into account the fact that leukocyturia may indicate tubular epithelial kidney injury. One of the possible diagnostic tests used to assess the dysfunction of renal tubules in the course of DMt2 is the measurement of UNGAL in a single urine sample and calculation of UNCR. In patients with leukocyturia, it has to be remembered that the presence of leukocytes in urine leads to increased UNGAL and UNCR. Moreover, in such patients, the correlations between UNCR and albuminuria, $\mathrm{HbA}_{1 \mathrm{c}}$ or age are diminished. In our opinion, there is a need for further prospective studies to validate the clinical usefulness of UNGAL and UNCR for the detection of kidney disease in DMt2, recruiting higher numbers of patients, and including those with sterile leukocyturia.

\section{Conflict of interest}

The authors declare no conflict of interest.

\section{References}

1. Saran R, Yi Li, Robinson B, et al. United States Renal Data System 2014 Annual Data Report. Epidemiology of kidney disease in the United States. Am J Kidney Dis 2015; 66: 1-306.

2. Fioretto P, Mauer M. Histopathology of diabetic nephropathy. Semi Nephrol 2007; 27: 195-207.

3. Fioretto P, Mauer M, Brocco E, et al. Patterns of renal injury in NIDDM patients with microalbuminuria. Diabetologia 1996; 39: 1569-76.

4. Haase M, Bellomo R, Haase-Fielitz A. Neutrophil gelatinase-associated lipocalin. Curr Opin Crit Care 2010; 16: 526-32.

5. Woo KS, Choi JL, Kim BR, Kim JE, An WS, Han JY. Urinary neutrophil gelatinase-associated lipocalin levels in com- 
parison with glomerular filtration rate for evaluation of renal function in patients with diabetic chronic kidney disease. Diabetes Metab J 2012; 36: 307-13.

6. Mishra J, Ma Q, Prada A, et al. Identification of neutrophil gelatinase-associated lipocalin as a novel early urinary biomarker for ischemic renal injury. J Am Soc Nephrol 2003; 14: 2534-43.

7. Matsa R, Ashley E, Sharma V, Walden AP, Keating L. Plasma and urine neutrophil gelatinase-associated lipocalin in the diagnosis of new onset acute kidney injury in critically ill patients. Crit Care 2014; 8: R137.

8. Ding $\mathrm{H}, \mathrm{He} \mathrm{Y}$, Li K, et al. Urinary neutrophil gelatinaseassociated lipocalin (NGAL) is an early biomarker for renal tubulointerstitial injury in IgA nephropathy. Clin Immunol 2007; 123: 227-34.

9. Rysz J, Banach M, Stolarek RA, et al. Serum metalloproteinases MMP-2, MMP-9 and metalloproteinase tissue inhibitors TIMP-1 and TIMP-2 in patients on hemodialysis. Int Urol Nephrol 2011; 43: 491-8.

10. Malyszko J, Malyszko JS, Kozminski P, et al. Possible relationship between neutrophil gelatinase-associated lipocalin, hepcidin, and inflammation in haemodialysed patients. Nephron Clin Pract 2010; 115: c268-75.

11. Cullen MR, Murray PT, Fitzgibbon MC. Establishment of a reference interval for urinary neutrophil gelatinaseassociated lipocalin. Ann Clin Biochem 2012; 49: 190-3.

12. Decavele AS, Dhondt L, De Buyzere ML, Delanghe JR. Increased urinary neutrophil gelatinase associated lipocalin in urinary tract infections and leukocyturia. Clin Chem Lab Med 2011; 49: 999-1003.

13. American Diabetes Association. Classification and diagnosis of diabetes. Diabetes Care 2015; 38: 8-16

14. Banach M, Aronow WS, Serban C, et al. Lipids, blood pressure and kidney update 2014. Pharmacol Res 2015; 95-96: 111-25.

15. Schulze BD. Urine diagnosis and leukocyturia. MMW Fortschr Med 2004; 146: 39-41.

16. Ding $\mathrm{H}, \mathrm{He} \mathrm{Y}$, Li K, et al. Urinary neutrophil gelatinase-associated lipocalin (NGAL) is an early biomarker for renal tubulointerstitial injury in IgA nephropathy. Clin Immunol 2007; 123: 227-34.

17. D’Amico G, Bazzi C. Urinary protein and enzyme excretion as markers of tubular damage. Curr Opin Nephrol Hypertens 2003; 12: 639-43.

18. Pavenstädt H, Kriz W, Kretzler M. Cell biology of the glomerular podocyte. Physiol Rev 2003; 83: 253-307.

19. Colantonio LD, Baber U, Banach M, et al. Contrasting cholesterol management guidelines for adults with CKD. J Am Soc Nephrol 2015; 26: 1173-80.

20. Sola D, Rossi L, Schianca GPC, et al. Sulfonylureas and their use in clinical practice. Arch Med Sci 2015; 11: 840-8.

21. Papaetis GS, Papakyriakou P, Panagiotou TN. Central obesity, type 2 diabetes and insulin: exploring a pathway full of thorns. Arch Med Sci 2015; 11: 463-82.

22. KDIGO Clinical Practice Guideline for the Evaluation and Management of Chronic Kidney Disease. Kidney Int 2013; 3: 1-150.

23. Bolignano D, Lacquaniti A, Coppolino G, et al. Neutrophil gelatinase-associated lipocalin as an early biomarker of nephropathy in diabetic patients. Kidney Blood Press Res 2009; 32: 91-8.

24. Nielsen SE, Reinhard H, Zdunek D, et al. Tubular markers are associated with decline in kidney function in proteinuric type 2 diabetic patients. Diabetes Res Clin Pract 2012; 97: 71-6.
25. Porrin E, Ruggenenti P, Mogensen CE, et al. Non-proteinuric pathways in loss of renal function in patients with type 2 diabetes. Lancet Diabetes Endocrinol 2015; 3: 382-91.

26. Wu J, Ding Y, Zhu C, et al. Urinary TNF-alpha and NGAL are correlated with the progression of nephropathy in patients with type 2 diabetes. Exp Ther Med 2013; 6: 1482-8.

27. Yang YH, He XJ, Chen SR, Wang L, Li EM, Xu LY. Changes of serum and urine neutrophil gelatinase-associated lipocalin in type-2 diabetic patients with nephropathy: one year observational follow-up study. Endocrine 2009; 36: 45-51.

28. Retnakaran R, Cull CA, Thorne K, Adler Al, Holman RR; UKPDS Study Group. Risk factors for renal dysfunction in type 2 diabetes: U.K. Prospective Diabetes Study 74. Diabetes 2006; 55: 1832-9. 